\title{
Nuovi outsider e forme di rappresentanza del lavoro parasubordinato e professionale in Italia
}

Andrea Ciarini*

\section{Mercato del lavoro e mutamenti della rappresentanza in Italia}

L'Italia è uno dei paesi europei in cui la membership sindacale si è mantenuta più alta in questi anni. Questa forza delle organizzazioni sindacali è tuttavia messa alla prova da difficoltà nella rappresentanza dei lavoratori più ai margini del mercato del lavoro, maggiormente sottoposti a fenomeni di instabilità occupazionale e in generale inquadrati in tipologie contrattuali atipiche, compresa una nutrita fascia di soggetti solo formalmente indipendenti ma nella sostanza dipendenti per la mancanza di garanzie collegate al lavoro standard: parasubordinati, collaboratori, lavoratori con partita Iva mono-committenti (Ranci, 2012; Carrieri e Treu, 2013; Mingione et al., 2014; Pugliese, 2014). Sull'emergere di questa nuova domanda di rappresentanza influiscono diversi fattori. In primo luogo va considerato il peggioramento delle condizioni di lavoro per larga parte del lavoro parasubordinato e anche professionale, un fenomeno relativamente recente e complicato dalla crisi economica. Rispetto ai primi anni del duemila quando gli studi segnalavano una crescita del lavoro autonomo terziario a più alta qualificazione - pur in presenza di forti divaricazioni interne quanto a redditi e insicurezza sociale (in particolare Fullin, 2004; Pavolini, 2012) - la crisi ha determinato un sostanziale peggioramento, con effetti negativi sul mercato dei servizi professionali. Come molte indagini hanno segnalato (Mingione et al., 2014; Pavolini, 2012; Cerea e Moiso, 2012; Reyneri e Pintaldi, 2013) questo peggioramento riguarda in particolare il gruppo dei collaboratori e dei professionisti con partita Iva

* Sapienza Università di Roma.

Sociologia del lavoro, n. 140/2015 
mono-committenti, andati ad ingrossare un eterogeneo gruppo spinto ai margini del mercato del lavoro, tra la limitata domanda di occupazioni terziarie qualificate e la scelta, più o meno obbligata, del lavoro professionale in alternativa al lavoro alle dipendenze.

Un secondo elemento da tenere in considerazione, collegato al primo, riguarda l'accesso alle tutele di welfare. Per i professionisti il problema delle prestazioni sociali ha molto a che fare con i limiti delle tutele ordinistiche, da un lato eccessivamente frammentate, dall'altro non in grado di assicurare coperture adeguate, se non al prezzo di un crescente aumento dei costi e dei contributi a carico degli iscritti. Anche i lavoratori parasubordinati hanno sostenuto negli ultimi anni un forte aumento del carico contributivo. Questa estensione, tuttavia, ad eccezione di limitate innovazioni come per esempio la una tantum per i collaboratori in caso di perdita del lavoro prevista dalla legge Fornero, non ha prodotto un effettivo miglioramento delle condizioni di protezione sociale, senza peraltro la possibilità di contare né su minimi contrattuali, né su tutele "dedicate" sul modello dei molti dispositivi reddito minimo ormai presenti in tutta Europa, eccezion fatta per l'Italia.

Tra deterioramento delle condizioni di lavoro e deboli protezioni di welfare non deve stupire che la rappresentanza di questi soggetti abbia iniziato ad entrare nel perimetro dell'organizzazione sindacale, sia quella tradizionalmente intesa ad opera dei maggiori sindacati confederali (su questo si veda Carrieri e Burroni, 2011; Gumbrell-McCormick e Hyman, 2013; Ambra, 2013; De Franceschi, Pulignano e Ortiz, 2014), sia in forme nuove, a partire da esperienze auto-organizzate che hanno iniziato a diffondersi in questi anni tra professionisti e parasubordinati.

Per molti aspetti l'auto-organizzazione è un fenomeno nuovo di cui ancora non si ha una esatta cognizione, né dal punto di vista teorico, né sul piano delle dimensioni concrete e delle strategie di rappresentanza. Per alcuni autori (in particolare Beccalli, Bacio e Meardi, 2014) cambiamenti in questa direzione riportano in primo piano logiche d'azione più simili al sindacalismo del XIX secolo, che non a quello verticale e istituzionalizzato del XX secolo. In questa chiave interpretativa che in parte richiama anche la tradizione del sindacalismo di mestiere, è interessante il richiamo all'auto-organizzazione come pratica agita da gruppi professionali autono$\mathrm{mi}$, soprattutto giovani con problemi di inserimento nel mercato del lavoro senza o con scarsa rappresentanza da parte dei sindacati e degli albi professionali: giovani architetti, ingegneri, archeologi, giornalisti, traduttori e interpreti, scrittori e lavoratori dello spettacolo.

Nelle pagine che seguono analizzeremo alcune di queste esperienze auto-organizzate: Iva sei partita (Architetti e Ingegneri), Strade (Sindacato Interpreti e traduttori), Acta (Associazione dei Consulenti del Terziario 
Avanzato) e Clap (Camere del Lavoro Autonomo e Precario). Si tratta di organizzazioni di rappresentanza di recente costituzione e sul piano della membership ancora a uno stadio iniziale, pur essendo alcune riuscite a dotarsi di strutture di gestione e partecipazione interna tra gli associati, oltre che di servizi dedicati a carattere mutualistico. Se si considera solo il numero degli aderenti siamo certamente di fronte a un fenomeno ancora marginale. Non è tuttavia solo questo il parametro con cui valutare l'impatto di queste organizzazioni sulla rappresentanza del lavoro professionale e parasubordinato. Vi è infatti uno spazio di rappresentanza più ampio - veicolato attraverso campagne web, mobilitazioni e servizi a carattere mutualistico - capace di aggregare molti più soggetti di quanti effettivamente associati, dando impulso alla costituzione di "coalizioni sociali" di ampia portata come vedremo. In effetti alcuni di questi gruppi hanno caratteristiche tali da farli sembrare veri e propri contro-movimenti polanyiani come ha scritto di recente Mingione (2014; vedi anche Borghi e Dorigatti, 2011; Burawoy, 2011; Rizza, 2011), tesi a contrastare gli effetti negativi indotti dall'acuirsi della crisi. Naturalmente non è solo questo il tratto distintivo di molte di queste esperienze. Sono molti i fattori concomitanti. Esso è tuttavia uno dei più interessanti da considerare, anche se molto controverso per il fatto di collocarsi spesso a metà strada tra il mutuo-aiuto, la rivendicazione di nuovi diritti sociali e il professionalismo. D'altra parte lo spettro delle richieste che emergono è molto vario, così come varie sono le strategie adottate e gli esiti delle mobilitazioni. A tutti gli effetti ci troviamo di fronte a un insieme di modelli di rappresentanza molto diversi tra loro e anche in via di consolidamento, tanto dall'essere suscettibili di cambiamenti, sia tra i nodi territoriali di medesime reti, sia nel tempo.

\section{Una rappresentanza autonoma in cerca di legittimazione}

Nello studio di queste reti emergenti di auto-rappresentanza ci sembra opportuno distinguere tra due grandi dimensioni. Da un lato il tipo e il grado di istituzionalizzazione raggiunto, da intendersi sia in riferimento alla vita associativa interna, sia nei rapporti con gli interlocutori esterni, siano essi organizzazioni sindacali o istituzioni pubbliche. Dall'altro, le strategie di rappresentanza, ovvero gli orientamenti e gli strumenti adottati nell'organizzare gli interessi dei soggetti rappresentati. Per quanto riguarda la prima di queste due dimensioni vi è in effetti una sostanziale distinzione tra chi ha conseguito, anche in tempi relativamente rapidi, una certa organizzazione interna e una rete di relazioni strutturate, spesso su singole vertenze aziendali con le organizzazioni sindacali (e anche con le istituzioni pubbliche come si vedrà più avanti) e chi, invece, si mantiene in 
uno stato più "magmatico" ma soprattutto più distante rispetto a una prospettiva di collaborazione e riconoscimento reciproco con il sindacato. Del gruppo di organizzazioni autonome più istituzionalizzate fanno parte l'associazione Iva Sei Partita, Strade e Acta. Di contro le Clap, pur non mancando di una organizzazione interna strutturata, fanno leva esplicitamente su un tipo di sindacalismo "attivista", costruito dal basso e fortemente orientato a saldare le singole vertenze sui luoghi di lavoro entro lo spazio più generale dei lavoratori precari della conoscenza. Strade, Acta e Iva sei partita, secondo caratteristiche diverse, sono organizzazioni autonome che hanno superato la fase inziale di spontaneismo organizzativo, in prima istanza veicolato a partire dal web.

Naturalmente l'istituzionalizzazione non è un attributo fisso. Ci sono diverse gradazioni e intensità di questo orientamento. Ci possono essere peraltro cambiamenti nel tempo che portano una organizzazione a strutturarsi diversamente. Teniamo conto del resto che parliamo di realtà spesso molto giovani, non consolidate e dunque soggette anche a mutamenti. In questa luce va letta l'esperienza di Iva sei partita, nata nel 2012 per tutelare architetti e ingegneri, ma già attiva dal 2010 attraverso la rete web. Iva sei partita nasce inizialmente su input di un ristretto gruppo di architetti e ingegneri per offrire uno spazio di interazione tra figure professionali che operano in sostanziale autonomia. Lo scambio di informazioni e la costituzione di una piattaforma comune sul web hanno dato inizio a un movimento eterogeneo al proprio interno e tuttavia capace di saldarsi intorno a istanze comuni: dal riconoscimento della condizione di subordinato nel caso di aderenti formalmente "professionisti", ma nella sostanza lavoratori subordinati per via della mono-committenza, alle vertenze sui canali di accesso alla libera professione e sul welfare di "categoria" (vedi meglio Ciarini, Di Nunzio e Pratelli, 2013). Rispetto ad altri casi che di seguito esamineremo Iva sei partita evidenzia un profilo certamente meno strutturato e probabilmente anche meno gerarchizzato quanto a organizzazione interna e reti di relazioni esterne. Ciò nonostante, non va sottovalutato l'avyio di un processo di collaborazione formale con le organizzazioni sindacali, con la Filcams Cgil in particolare in occasione del rinnovo del Contratto Collettivo Nazionale degli studi professionali. Anche grazie a questa collaborazione per la prima volta sono state inserite nel contratto tutele contrattuali per praticanti, partite iva e collaboratori. Alla stessa stregua è iniziato un percorso di istituzionalizzazione presso gli ordini professionali degli architetti e ingegneri. Nel caso del nodo romano dalla fase più spontaneista, nata intorno al web, l'organizzazione ha scelto di insediare propri rappresentanti nel sistema ordinistico, passando a una azione di rivendicazione formale su specifici punti riguardanti la professione, ancorché comuni a molta parte del lavoro professionale. Il riferimento va qui in particolare al contrasto verso l'aumento delle aliquote contributive 
minime per la previdenza, cresciute solo nel 2012 del 30\% (per effetto della riforma previdenziale dello stesso anno) e la ridefinizione dei parametri che regolano i rendimenti previdenziali, con il pieno regime del metodo di calcolo contributivo.

Le scelte compiute in questa fase sembrano segnare un deciso passaggio verso rivendicazioni a carattere professionale. Questo non significa però che il modello di rappresentanza prescelto sia circoscritto a questo ambito. Proprio in considerazione della centralità assunta dalle campagne sul welfare l'azione di Iva sei partita è andata allargandosi a questioni "sociali" che interessano da vicino tutto il mondo del lavoro parasubordinato e parte di quello professionale più esposto ai problemi di intermittenza lavorativa e vulnerabilità sociale (vedi meglio prossimo paragrafo). Da questo punto di vista la tendenza all'istituzionalizzazione che si rileva ha caratteristiche tali da non configurare un semplice passaggio verso vertenze dentro la professione. In realtà c'è un mescolarsi di questioni sociali e richieste contrattuali, in un intreccio tra entrambi i piani, anche se a partire da una accentuata dimensione professionale della rappresentanza, quale inequivocabilmente è per ingegneri e architetti.

Diverso in questo senso è il modello scelto da Strade, la seconda delle organizzazioni più tendenti alla istituzionalizzazione. Va detto anzitutto che la fondazione di Strade risale al 2012, dunque recente, a seguito tuttavia dell'esperienza maturata in otto anni come sezione tematica del Sindacato Nazionale Scrittori (SNS). Già questo è un primo tratto che allontana Strade da Iva sei partita, ancor più se si considera che nel 2012, è stato siglato un protocollo di intesa con il Sindacato Lavoratori della Conoscenza della Cgil, prefigurando dunque una forte collaborazione istituzionalizzata con il sindacato.

Attualmente gli iscritti ordinari a Strade sono 250, più una ventina di associati che ancora non hanno maturato i requisiti per l'iscrizione ordinaria, la quale sola dà diritto alla partecipazione attiva alle cariche elettive e alla fruizione di servizi interni. Il riconoscimento come soci ordinari è vincolato al rispetto di alcuni requisiti, il principale dei quali è la dimostrazione di un certo numero di prodotti editoriali eseguiti. In assenza di un ordine professionale di riferimento, è questo un elemento interessante per il fatto di costituire un vincolo alla partecipazione "attiva" che di solito rientra nelle prerogative degli ordini professionali. D'altra parte è anche questo il segno di una certa tendenza alla istituzionalizzazione che si riscontra in questa associazione, autonoma e pur tuttavia bene identificabile con una specifica professione. L'attività sindacale di Strade è nei fatti incentrata sulla tutela di un gruppo di professionisti qualificati che soffrono una condizione di particolare svantaggio nel mercato del lavoro, per il fatto di essere sottoposti a una disciplina contrattuale, quella del diritto d'autore, fortemente sbilanciata in favore 
dei committenti. Traduttori e interpreti sono professionisti tendenzialmente pluri-committenti e dotati di un tariffario. Il problema sono i bassi compensi dovuti alle deroghe ampiamente tollerate. Sul piano dell'azione di rappresentanza la collaborazione con la Cgil verte proprio su questo, ovvero sul riconoscimento di diritti contrattuali non derogabili e sulla richiesta prestazioni di welfare aggiuntive, in primis la maternità e l'assistenza integrativa.

L'assenza di tutele di welfare è uno dei temi più sentiti dagli aderenti a Strade, come a molte altre organizzazioni autonome di professionisti e parasubordinati. In questo caso però la soluzione individuata ha sue proprie specificità che in parte sono dovute a questa stretta relazione con una categoria sindacale. I soci di Strade hanno infatti la possibilità di fruire della tessera Slc-Cgil e di una serie di servizi forniti dalla categoria. Detto questo, vi sono prestazioni organizzate anche in forma autonoma, su base mutualistica, in particolare per quello che riguarda la sanità integrativa. L'assicurazione volontaria stipulata con la società di mutuo soccorso "Insieme Salute" garantisce ai soci ordinari diverse prestazioni, da un sussidio integrativo giornaliero in caso di malattia, a un assegno di gravidanza, fino al rimborso spese per prestazioni ospedaliere e specialistiche. Vi è infine la possibilità di vedersi riconosciute delle somme una tantum in caso di infortunio o perdita della autosufficienza. Questi servizi come già accennato sono garantiti ai soli soci ordinari, coloro cioè che non solo versano le quote associative ma che anche rispettano determinati requisiti sul piano delle professionalità acquisite. Sulla formula mutualistica ritorneremo più avanti nelle conclusioni. Qui ci basti dire che si tratta di una scelta che sta dentro una idea di mutualità a base professionale circoscritta sulla base di requisiti formali.

In una accezione diversa il richiamo alla mutualità è presente in molte altre formazioni auto-organizzate, ad esempio Acta, la terza delle organizzazioni autonome qui analizzate che hanno superato la fase spontaneista. Il bacino di riferimento di Acta, fondata nel 2004 a Milano, è un insieme molto più articolato di professionisti e lavoratori autonomi rispetto a Strade: formatori, informatici, creativi della comunicazione, designer, traduttori, ricercatori, fotografi, consulenti alle imprese, collaboratori a progetto, mono o pluricommittenti. Siamo a pieno titolo nel vasto campo del cosiddetto terziario avanzato o del "lavoro autonomo di seconda generazione" (Bologna e Fumagalli, 1997) - come peraltro esplicitamente dichiarato nel Manifesto dell'organizzazione. Vale a dire figure professionali autonome nuove, non regolamentate da ordini o albi professionali, emerse via via per effetto dei processi di esternalizzazione aziendali o interni alle pubbliche amministrazioni. Acta nasce espressamente per rappresentare questo variegato mondo dei servizi alle imprese e più in generale dei lavoratori della conoscenza, secondo una idea di rappresentanza che è andata 
nel tempo combinando diversi strumenti. La sua organizzazione interna fa capo a strutture di governo nazionali (Assemblea dei soci, Consiglio direttivo, Commissioni di studio), gruppi di lavoro su singole attività di rilievo per l'associazione (comunicazione, servizi agli associati, proposte politiche) e aree territoriali, al momento tre: Lombardia, Toscana, Lazio. A ciascuna area territoriale viene demandato il compito di istruire l'attività di rappresentanza e una serie abbastanza ampia di servizi per gli associati che vanno da convenzioni per la copertura sanitaria mutualistica (come per l'associazione Strade la scelta è ricaduta sulla mutua "Salute Insieme"), la previdenza complementare, l'acquisto a prezzi agevolati di buoni pasto, la consulenza legale e fiscale, fino a corsi di formazione e in ultimo la possibilità di usufruire di spazi di coworking a tariffe vantaggiose. Ad oggi il numero degli iscritti è pari a circa 3000 unità, più 400 soci paganti che partecipano attivamente alla vita dell'associazione. Ciascuno dei nodi territoriali ha sue proprie specificità, date prima di tutto dal bacino di utenza. In Lombardia è prevalente il lavoro di consulenza alle imprese, di contro nel caso del nodo romano, l'attività si mostra più orientata alla rappresentanza del lavoro autonomo e professionale di supporto alle pubbliche amministrazioni. E questo non deve stupire in considerazione dell'ampio bacino di lavoro precario cresciuto intorno all'amministrazione pubblica. Tra il 2014 e il 2015 si sono aggiunti due nuovi nodi regionali: Veneto e Friuli-Venezia Giulia, mentre sono in via di costituzione i primi due del Mezzogiorno: Sicilia e Campania.

Accanto a queste molteplici attività di servizio l'azione di rappresentanza si è andata sviluppando in due direzioni principali. Da un lato l'individuazione di spazi di collaborazione con le organizzazioni sindacali, specie i patronati, e con le istituzioni per quello che riguarda la formazione professionale, uno degli ambiti di intervento prioritari per Acta. Dall'altro, l'intensificarsi dei rapporti con altre organizzazioni autonome di professionisti e parasubordinati su campagne per il riconoscimento di diritti sociali che esulano dall'ambito strettamente "professionale". Nel caso del nodo romano, costituito nel 2010, la collaborazione con le istituzioni ha trovato una prima concreta iniziativa sostenuta dalla Provincia in un corso di formazione ad hoc per futuri lavoratori autonomi del terziario avanzato. A tale proposito va anche ricordato che la sede del nodo romano è stata messa a disposizione dalla medesima Provincia. Sempre in tema di collaborazione non va dimenticata la relazione che si è venuta a configurare con i patronati dei sindacati confederali, questo in particolare nelle attività di servizio svolte sui temi legati alla previdenza e ai diritti sociali connessi all'allargamento, ancorché parziale, degli ammortizzatori sociali dopo le ultime riforme. Il riferimento va naturalmente qui alla una tantum per i co.co.pro prevista dalla riforma Fornero e da ultimo alla indennità - sperimentale - Discol per i collaboratori introdotta con il Jobs Act. 
Per quello che riguarda i rapporti con altre organizzazioni autonome, siamo di fronte a un campo emergente che segna un certo slittamento nella strategia di rappresentanza. L'azione di ACTA è infatti andata saldandosi con quella di altre organizzazioni autonome intorno a rivendicazioni sociali di carattere universalistico, pur continuando a rivendicare la specificità del lavoro autonomo e l'assoluta indisponibilità a una parificazione con il lavoro dipendente standard.

Molte delle più recenti campagne di Acta, da quella per la parificazione contributiva e fiscale tra dipendenti, commercianti, professionisti e iscritti alla gestione separata Inps (bacino entro cui si trova la gran parte dei soggetti rappresentati), alla richiesta di revisione del sistema previdenziale contro le iniquità generate dal metodo contributivo 1 , fino alle questioni del sostegno del reddito e della maternità, stanno a pieno titolo dentro rivendicazioni universaliste. Dentro "questioni sociali" fortemente incardinate in una prospettiva di welfare universalista meno ancorato alle tradizionali logiche categoriali e assicurative. Campagne di questo tipo riflettono un mutamento di strategia indotto anche dal peggioramento delle prospettive lavorative di molti collaboratori e parasubordinati in conseguenza della crisi, a fronte di continui aggravi sulla gestione separata in termini di aliquote contributive senza adeguate prestazioni sociali in cambio. Il terziario avanzato è in effetti, come abbiamo già sottolineato all'inizio, investito da un generale scadimento delle condizioni del mercato, senza un sistema di tutele in grado di controbilanciare l'insorgere di fenomeni di vulnerabilità sociale. Non deve stupire a questo proposito che Acta, almeno in alcune sue componenti interne, sia entrata a fare parte di coalizioni del lavoro professionale che pongono fortemente questioni intrecciate alla riforma in senso universalista del welfare.

\section{L'auto-organizzazione dei professionisti come movimento dei lavo- ratori precari}

Il tratto originale di questo passaggio, che coinvolge va detto soprattutto il nodo romano, coincide anche con l'avvio di una mobilitazione collettiva su più vasta scala intorno alla denominata "Coalizione 27 Aprile", una coalizione sociale costituitasi nel 2015 di cui fanno parte oltre 19 organiz-

1. La campagna per la riforma previdenziale sostiene il superamento del metodo contributivo verso un sistema multi-pilastro fondato su una pensione di base, aggiuntiva a quella contributiva, legata al numero degli anni lavorati, indipendentemente dai contributi versati e dalla tipologia di lavoro svolto. Insomma una pensione di base universale, tesa a controbilanciare il rischio di rendimenti estremamente bassi che si profilano dall'applicazione integrale del metodo di calcolo contributivo. Della proposta fa parte anche il versamento di contributi figurativi a copertura degli impegni di cura familiare. 
zazioni autonome, tra cui le Clap, Iva sei Partita, più altre organizzazioni di professionisti e para-subordinati, tra cui giornalisti, archivisti, avvocati, geometri, ricercatori precari, su una piattaforma rivendicativa orientata soprattutto al riconoscimento di diritti universali: dalla pensione di base universale, alla estensione universale delle indennità di malattia, maternità, e ammortizzatori sociali, compreso un reddito di base sul modello degli schemi di reddito minimo garantito.

Le Camere Autonome del Lavoro Precario (Clap), di cui ci occuperemo nel prosieguo sono state tra i primi promotori di questa coalizione sociale. Le Clap nascono a Roma nel 2014 da una esperienza di integrazione tra sportelli, comitati e esperienze di auto-organizzazione di precari per offrire spazi di rappresentanza a un ampio gruppo di lavoratori autonomi a bassi redditi, atipici, partite Iva, collaboratori disoccupati, stagisti e tirocinanti. In questo senso l'adesione alla coalizione non solo è nella strategia di rappresentanza promossa. È a tutti gli effetti un attributo fondante, generativo dell'azione sindacale per come essa è stata intesa.

Attualmente i nodi della rete si distribuiscono su quattro sedi territoriali nei municipi di Roma. Al loro interno, trova spazio una attività di assistenza e consulenza in materia previdenziale, fiscale, normativa sia di taglio generale, sia finalizzata a contrastare, attraverso vertenze individuali, l'utilizzo improprio dei contratti a collaborazione o delle partite Iva. Questa attività di servizio, si salda con una azione di rappresentanza tesa a sostenere fortemente l'auto-organizzazione dei lavoratori autonomi in ogni vertenza aziendale o territoriale. Le vertenze attivate (i lavoratori dei servizi in sub-appalto nella logistica, i precari del gruppo La7, i lavoratori dell'azienda di trasporto pubblico locale Atac) si rifanno espressamente a mobilitazioni dal basso, in cui sono direttamente coinvolti, come attivisti, $\mathrm{i}$ lavoratori organizzati. Ad esse si sono aggiunte in poco meno di due anni mobilitazioni riguardanti i lavoratori con partita Iva del settore sanitario, centri commerciali e dei servizi sociali.

Il tipo di mutualismo che qui emerge è per molti aspetti diverso da quello che caratterizza altre realtà auto-organizzate. Più che ai fini dell'erogazione di prestazioni e servizi negati sul piano contrattuale, siamo di fronte a un "mutualismo di resistenza" finalizzato a rompere l'isolamento che caratterizza molta parte del lavoro autonomo e parasubordinato. Inchieste, campagne comunicative, picchetti fanno parte di questo mutualismo, inteso come alfabetizzazione alla pratica sindacale in prima persona. Lontano da modelli di rappresentanza su base categoriale e professionale, il richiamo all'auto-organizzazione, ma anche al riconoscimento dei diritti sul lavoro e sociali, su tutti quelli legati al reddito di base, prefigura un doppio binario di rappresentanza. Il primo, teso a organizzare sia il lavoro poco sindacalizzato e più vulnerabile, sia quello tradizionalmente stabile ma suscettibile di precarizzazione (come nel caso dei lavoratori 
del trasporto pubblico locale). Il secondo livello, di portata più generale, è incentrato su campagne più trasversali per il riconoscimento di diritti sociali universali dentro coalizioni sociali larghe, come la rete 27 Aprile. Che le Clap facciano parte di questa coalizione è abbastanza comprensibile. Più sorprendente, se si guarda agli insediamenti sociali tradizionali del lavoro autonomo, è che di essa siano entrati a fare parte anche Iva sei partita e Acta.

\section{Considerazioni conclusive. Lavoro e rivendicazioni "sociali" nella rappresentanza del lavoro autonomo e professionale}

La diffusione di queste reti auto-organizzate, pur nella loro varietà interna, ci mette di fronte a cambiamenti che iniziano ad arricchire il versante della rappresentanza. Certo nella maggior parte dei casi si tratta di organizzazioni non necessariamente destinate a legittimarsi. Sono tuttavia indicative, queste esperienze, di una domanda di rappresentanza nuova e molto più articolata rispetto al passato che preme tanto sulle istituzioni, quanto sulle grandi organizzazioni sociali che non a caso hanno iniziato a modificare gli assi della contrattazione e l'organizzazione interna. Va detto da questo punto di vista che abbiamo di fronte non semplici segmenti di lavoratori marginali come almeno fino al più recente passato si intendeva il problema dei non rappresentati. Tra questi vi è oggi una componente non minoritaria di lavoratori e professionisti del terziario avanzato assai più esposti che in passato ai problemi della mancanza di reddito e di tutele nel welfare.

I problemi che qui si intrecciano sono vari, così come vario è il campo delle relazioni con il sistema della rappresentanza sindacale. Alcune di queste organizzazioni come abbiamo visto tendono a ricercare forme di collaborazione con i sindacati su singole vertenze, siano esse relative al "mestiere" o questioni più generali, altre a stressare la parte più direttamente riconducibile alla ricerca di spazi di auto-organizzazione tanto dalle istituzioni, quanto dai sindacati tradizionali, altre ancora a combinare alcuni o tutti questi elementi. Alcune perseguono obiettivi più generali non riconducibili a una specifica professione ma a una prospettiva più generale, altre rimangono dentro l'azione vertenziale per il miglioramento dei trattamenti e delle protezioni sociali di un gruppo professionale ben definito.

Allo stesso modo anche le logiche d'azione sono differenti, sebbene in molte di esse emerga il riferimento a un agire autonomo e di mutuo-aiuto come tratto preponderante della rappresentanza. Questo mutuo-aiuto trova una diversa declinazione a seconda che si tratti di organizzazioni strutturate e identificate con una professione specifica oppure che la platea 
dei rappresentati sia più varia. Nel primo caso (si veda in particolare il caso di Strade) il mutualismo è la risposta autonoma e auto-organizzata a un deficit di tutele nel welfare, pubblico e di categoria. Diversamente nel caso delle reti meno istituzionalizzate ma anche più orientate ad agire nello spazio più generale del movimento dei lavoratori precari, l'accezione mutualistica ricorda più una dimensione di "lotta" (non la tutela associativa su base volontaria) che pure rientra sempre nella trazione di queste pratiche. In questo ambito l'auto-organizzazione si salda con pratiche di rappresentanza "attiviste", volte a costruire coalizioni le più larghe possibile per ottenere il riconoscimento di diritti sociali connessi al welfare soprattutto. Queste coalizioni non necessariamente sfociano nella costruzione di rapporti di collaborazione formale con le grandi organizzazioni sindacali. Parimenti distante è l'orizzonte della contrattazione, come mezzo attraverso il quale costruire progressivamente sistemi di tutele dedicate. Siamo piuttosto nello sconfinamento verso un tipo di sindacalismo basato su mobilitazioni di base, campagne e rivendicazioni di portata generale (il reddito di base, i diritti al welfare) a partire da singole vertenze che vedono coinvolti i lavoratori più vulnerabili, anche del terziario avanzato.

Le organizzazioni autonome che abbiamo esaminato si collocano su un range di posizioni molto articolato, quanto a grado istituzionalizzazione e strategie di rappresentanza. Vi è tuttavia un elemento comune un po' a tutte che a nostro avviso spiega la centralità delle mobilitazioni intorno alle "questioni sociali". Il fatto è che di fonte al peggioramento del mercato e alla crisi (che ha scaricato i suoi effetti negativi anche sul lavoro autonomo) la debolezza o l'assenza tout court di tutele di welfare accomuna la condizione di tutti questi lavoratori, professionisti o parasubordinati. In chiave di rappresentanza si tratta di un elemento da non sottovalutare. Esso segnala quasi un ribaltamento di prospettiva rispetto allo stato - tradizionale - dei rapporti tra lavoro dipendente e lavoro autonomo. Come è noto (Paci, 1993; Pennacchi, 1994; Ranci, 2012), almeno fino a tutti gli anni Ottanta e Novanta era sul lavoro dipendente che gravavano la gran parte degli oneri contributivi e fiscali delle politiche sociali e previdenziali, a tutto vantaggio del lavoro autonomo, nei fatti favorito dall'aumento delle prestazioni sociali e dall'allargamento in senso universalista di parti del sistema di welfare, senza un pari corrispettivo in termini di contributi sociali e previdenziali. Rispetto a questo non troppo lontano passato la condizione del lavoro parasubordinato è molto diversa. Come è stato osservato da Ranci (2012; si veda anche Paci, 1992) in merito a quel sistema di reciproche opposizioni ma anche convenienze nascoste che a lungo ha caratterizzato il rapporto tra dipendenti e autonomi - ai lavoratori dipendenti il peso del carico fiscale ma in cambio di elevate garanzie sul posto di lavoro e nel welfare, agli autonomi (relativi) minori livelli di prestazioni sociali, ma a fronte di un tacito regime di tolleranza fiscale - lo scenario 
che abbiamo di fronte è sensibilmente diverso. Se è vero infatti che la legislazione sul lavoro e in materia di welfare ha posto fine progressivamente a queste pratiche di «incorporazione nel welfare pubblico a basso costo» (ivi, p. 27), bisogna riconoscere che l'aumento del carico contributivo sul lavoro professionale e parasubordinato non ha prodotto un effettivo miglioramento delle condizioni di protezione sociale per questi lavoratori, nella sostanza ancora ai margini delle tutele assicurative, senza peraltro la possibilità di contare su minimi contrattuali o una rete universale di protezione sociale.

La mancanza di diritti sociali universali, siano essi legati alla maternità o al sostegno del reddito, è insomma un problema trasversale a parasubordinati e professionisti. Non appare un caso in questo senso che sulla costituzione di alleanze e coalizioni sociali (si veda a tale proposito la coalizione 27 Aprile di cui abbiamo dato conto) si ritrovino anche organizzazioni che agiscono in uno spazio di rappresentanza tutto interno a una specifica professione, come per esempio Iva sei partita. Questo aspetto è tanto più significativo se si considera il passaggio che questa organizzazione ha fatto più di recente dentro il sistema ordinistico di architetti e ingegneri. Pur avendo scelto di concentrare la grande parte delle risorse organizzative in questa direzione, in sé più circoscritta a una tutela "esclusiva" dei professionisti che si rappresentano, ciò non di meno non è mancata l'adesione a una coalizione sociale che pone tra i suoi obiettivi l'estensione universale del welfare su più piani, dalla maternità, alla previdenza, fino a forme di reddito minimo. Certo è una novità che su rivendicazioni di questa portata si trovino organizzazioni come queste, da chi tutela professionisti appartenenti a ordini a chi, come Acta, punta invece a rappresentare il più variegato mondo dei lavoratori professionali del terziario avanzato.

\section{Riferimenti bibliografici}

Ambra M.C. (2013). Modelli di rappresentanza sindacale nella società postindustriale. Come i sindacati si stanno ri-organizzando. Quaderni di Rassegna sindacale - Lavori, 4: 75-94.

Beccalli B., Bacio M., Meardi G.(2014). Varietà delle forme di rappresentanza di lavoratori e cittadini: verso una analisi comparata e internazionale. Quaderni di Rassegna sindacale - Lavori, n. 1: 11-25.

Bologna S., Fumagalli A., a cura di (1997). Il lavoro autonomo di seconda generazione. Milano: Feltrinelli.

Borghi V., Dorigatti L. (2011). Trasformazioni del lavoro, globalizzazione e ricerca sociale: piste di esplorazione per rinnovare la difesa del lavoro. Sociologia del Lavoro, 3: 32-48. DOI: 10.3280/SL2011-123003

Burawoy M. (2011). La svolta pubblica: dal processo lavorativo al movimento operaio. Sociologia del Lavoro, 3: 15-31. DOI: 10.3280/SL2011-123002 
Burroni L., Carrieri M. (2011). Bargaining for social rights. BARSORI country report Italy.

Carrieri M., Treu T., a cura di (2013). Verso nuove relazioni industriali. Bologna: il Mulino.

Cerea S., Moiso V. (2012). Le associazioni e le forme di rappresentanza. In: Ranci C., a cura di, Partive Iva. Il lavoro autonomo nella crisi italiana. Bologna: il Mulino.

Ciarini A., Di Nunzio D., Pratelli C. (2013). Nuove forme di autorganizzazione in Italia. Quaderni di Rassegna Sindacale - Lavori, 4: 133-152.

De Franceschi F., Pulignano V., Ortiz L. (2014). Il lavoro precario e le strutture sindacali. Le strategie adottate dai sindacati italiani e spagnoli. Quaderni di Rassegna Sindacale - Lavori, 1: 161-195.

Fullin M. G. (2004). Vivere l'instabilità del lavoro. Bologna: il Mulino.

Gumbrell-McCormick R., Hyman R. (2013). Trade Unions in Western Europe. Hard Times, Hard Choices. Oxford: Oxford University Press.

Mingione E., Andreotti A., Benassi D., Borghi P., Cavalca G., Fellini I. (2014). Le organizzazioni sociali e i giovani professionisti nell'area milanese. Quaderni di Rassegna Sindacale - Lavori, 1: 123-140.

Paci M. (1992). Il mutamento della struttura sociale in Italia. Bologna: il Mulino. Pavolini E. (2012). Gli indipendenti fra ceto e classe. In: Ranci C., a cura di, Partite Iva. Il lavoro autonomo nella crisi italiana. Bologna: il Mulino.

Pavolini E., Ascoli U., Mirabile M. L., a cura di (2013). Tempi moderni. Il welfare nelle aziende in Italia. Bologna: il Mulino.

Pennacchi L., a cura di (1994). Le ragioni dell'equità: principi e politiche per il futuro dello stato sociale. Bari: Dedalo.

Pugliese E. (2014). Presentazione. La varietà delle esperienze e i loro possibili impatti. Quaderni di Rassegna sindacale - Lavori, n. 1: 7-10.

Ranci C., a cura di (2012). Partite Iva. Il lavoro autonomo nella crisi italiana. Bologna: il Mulino.

Reyneri E., Pintaldi F. (2013). Dieci domande su un mercato del lavoro in crisi. Bologna: il Mulino.

Rizza R. (2011). Partecipazione e lavoro. Sindacati, movimenti sociali e forme di auto-organizzazione: un'introduzione. Sociologia del Lavoro, 3: 7-11. DOI: 10.3280/SL2011-123001 